\title{
Fully-Integrated CMOS LDO Regulator Based on an Embedded Current-Mode Capacitor Multiplier
}

\author{
A. Saberkari, Member, IEEE, H. Martínez, Member, IEEE, R. Fathipour and E. Alarcón, Member, IEEE
}

\begin{abstract}
This article presents a fully-integrated CMOS output-capacitorless low-dropout voltage regulator (LDO). A capacitor multiplier based on a current-mode amplifier is embedded into the error amplifier of the aforementioned LDO to simultaneously enhance the dynamic specifications to load variations, stability by pole splitting, and power saving. The proposed LDO topology is designed and post simulated using a $0.35 \mu \mathrm{m}$ CMOS process to deliver a load current between 0-100 $\mathrm{mA}$. The dropout voltage of the $\mathrm{LDO}$ is set to $200 \mathrm{mV}$ for $2-3.5 \mathrm{~V}$ input voltage. A final benchmark comparison considering all relevant performance metrics is presented.
\end{abstract}

Keywords - Current-Mode Amplifier, Embedded Capacitor Multiplier, Error Amplifier, Fast Transient, Low-Dropout Regulator, Output-Capacitorless.

\section{INTRODUCCIÓN}

L OS MODERNOS dispositivos electrónicos portátiles como teléfonos móviles y PDAs necesitan, irremediablemente, sistemas de gestión de energía eficientes para reducir la potencia en modo reposo (standby) e incrementar, como consecuencia, la vida de sus baterías. Sin embargo, generalmente, los consumos de baja potencia implican menor velocidad y reducción del ancho de banda del sistema electrónico en cuestión. Por lo tanto, la mejora de la velocidad y de la respuesta transitoria en diseños de baja potencia debería ser un aspecto clave en el diseño de este tipo de sistemas de bajo consumo. Los reguladores de baja tensión de dropout ( $L D O$ regulators) son una opción ideal y ampliamente utilizada en multitud de circuitos integrados para gestión de potencia. Además, dichos reguladores son utilizados como postreguladores a la salida de convertidores conmutados de alto rendimiento, puesto que los primeros pueden asegurar un buen ancho de banda y una regulación fiable con bajo ruido y bajos rizados en las tensiones de alimentación finales para cargas de RF y analógicas sensibles al ruido [1].

La estructura típica de un regulador LDO se muestra en la Fig. 1. Ésta consiste en un amplificador de error que detecta el error entre la referencia y la tensión de salida, un transistor de paso $\left(M_{p}\right)$, controlado por el amplificador de error, que regula el flujo de corriente hacia la carga, una red de realimentación (resistores $R_{F 1}$ y $R_{F 2}$ ), y un condensador de salida $\left(C_{\text {out }}\right)$.

A. Saberkari, Microelectronics Research Lab., Department of Electrical Engineering, University of Guilan, Rasht, Iran, a_saberkari@guilan.ac.ir

H. Martínez, Department of Electronics Engineering, Technical University of Catalonia, Barcelona, Spain, herminio.martinez@upc.edu

R. Fathipour, Microelectronics Research Lab., Department of Electrical Engineering, University of Guilan, Rasht, Iran. a_saberkari@guilan.ac.ir

E. Alarcón, Department of Electronics Engineering, Technical University of Catalonia, Barcelona, Spain, eduard.alarcon@upc.edu
Por requerimientos de estabilidad, la mayoría de reguladores LDO convencionales integrados en forma de chip utilizan condensadores externos (off-chip) de gran tamaño del orden de centenares de $n F$ o, incluso, algún $\mu F$ [1-6]. Ahora bien, como condensadores de elevado valor no pueden ser integrados en forma de chip (on-chip), la obtención de reguladores LDOs sin necesidad de condensador de salida es primordial para aplicaciones SoC. Sin embargo, el simple hecho de eliminar el condensador de salida para obtener implementaciones completamente integrada on-chip lleva consigo algunos retos importantes, como es la degradación de las prestaciones dinámicas del LDO frente a transitorios de carga rápidos.

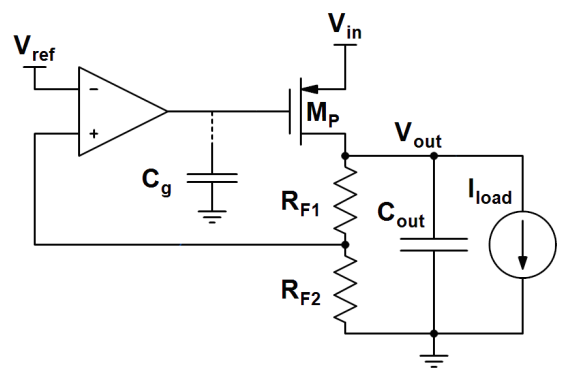

Figura. 1. Estructura típica de un regulador LDO.

Trabajos previos han presentado diferentes soluciones al respecto. En [7], por ejemplo, un regulador LDO de respuesta transitoria rápida con un condensador de salida on-chip de 600 $p F$ ha sido presentado. Sin embargo, la implementación de este valor de capacidad requiere una considerable área de silicio en el chip. Además, el circuito consume una importante corriente de reposo (quiescent), del orden de $6 m A$ y, por tanto, no es apropiado para diseños de bajo consumo. Para conseguir una adecuada respuesta transitoria, en [8] se utiliza un multiplicador de capacidad como etapa separada entre la salida del LDO y la puerta del transistor de paso, proporcionando así un camino rápido $\mathrm{y}$, consecuentemente, mejorar las prestaciones dinámicas del regulador LDO. Sin embargo, esta etapa multiplicadora de capacidad añade un consumo de potencia adicional al circuito sin beneficios importantes sobre el lazo de realimentación principal. En [9] la técnica conocida como compensación nested-Miller es utilizada, con condensadores del orden de $50 \mathrm{pF}$. Esto proporciona un buen margen de fase $\mathrm{y}$ acota el sobreimpulso en la respuesta transitoria. Sin embargo, como ya se ha mencionado anteriormente, estos valores de condensador ocupan elevadas áreas de silicio en el chip. Además, la tensión de salida varía de forma significativa cuando la corriente de carga se modifica. Los LDOs basados en la técnica flipped voltage follower (FVF) fueron introducidos en [10-12], puesto que pueden operar a tensiones muy reducidas, permitiendo, a su vez, una elevada capacidad de 
corriente de carga y proveyendo baja impedancia de salida. Sin embargo, estos reguladores adolecen de deficientes regulaciones de línea y de carga, y requieren la necesidad de tener una corriente de carga mínima que asegure la estabilidad del circuito.

El presente artículo presenta un regulador LDO sin condensador de salida en el que se incluye, en el propio amplificador de error, un multiplicador de capacidad basado en un amplificador en modo corriente. La inclusión en el citado amplificador de error proporciona un ahorro de potencia y, simultáneamente, mejora las prestaciones dinámicas del regulador en cuanto a transitorios de carga. La Sección 2 describe la red de separación de polos que mejora la velocidad y enriquece las prestaciones dinámicas del LDO. El LDO propuesto basado en el multiplicador en modo corriente incluido en el diseño se introduce en la Sección 3. Finalmente, la caracterización del circuito y las principales conclusiones del artículo son expuestas, respectivamente, en las Secciones 4 y 5.

\section{RED PARA LA MEJORA DE LA VELOCIDAD Y SEPARACIÓN DE POLOS}

El hecho de utilizar un condensador externo de gran valor en la salida de un regulador LDO, proporciona un cero en el semiplano izquierdo (LHP zero) en la función de transferencia en lazo abierto del sistema, que es debido a la resistencia electroestática (ESR) no despreciable de dicho condensador. Dicho cero acarrea una mejora en la estabilidad del circuito [13], y, además, reduce los rizados (ripples) de salida, $\Delta V_{\text {out }}$, debidos a los transitorios de carga y de línea. Cuando la corriente de carga cambia rápidamente de 0 a $I_{\max }$, debido a la limitación de ancho de banda, la corriente a través del transistor de paso estará inicialmente fijada y, por tanto, la caída de la tensión de salida está dada por [8]:

$$
\Delta V_{\text {out }} \cong \frac{I_{\max } \Delta t}{C_{\text {out }}},
$$

donde $\Delta t$ es el intervalo de tiempo que el lazo tarda en reaccionar. Como se observa, $\Delta V_{\text {out }}$ es inversamente proporcional a $C_{\text {out }} \mathrm{y}$, como consecuencia, al decrecer el valor del condensador de salida, dicho rizado se incrementa. Además, las respectivas posiciones de los polos en la puerta del transistor de paso $M_{P}$ (polo $\left.P_{1}\right)$, y en el nodo de salida del LDO $\left(P_{2}\right)$ están dados, respectivamente, por:

$$
P_{1} \approx \frac{-1}{R_{o, E A} C_{g}} \text { y } P_{2} \approx \frac{-1}{R_{\text {out }} C_{\text {out }}},
$$

donde $R_{o, E A}$ es la resistencia de salida del amplificador de error, $C_{g}$ es la capacidad total en la puerta del transistor $M_{P}$, que consiste en la capacidad formada por efecto Miller debida a la capacidad puerta-drenador de $M_{P}$ multiplicada por el factor $\mathrm{g}_{m p} R_{\text {out }}$, siendo $R_{\text {out }}=r_{\text {op }} \|\left(R_{F 1}+R_{F 2}\right) \approx r_{o p}$ la resistencia de salida equivalente del LDO, donde $g_{m p}$ y $r_{o p}$ son las transconductancia y resistencia de salida del transistor $M_{P}$, respectivamente. Las respectivas posiciones de estos dos polos dependen de la carga y esta dependencia es especialmente más significativa en el polo de salida $P_{2}$. Como consecuencia, la distancia entre estos dos polos decrece especialmente para pequeñas corrientes de salida, haciendo que el circuito tienda a la inestabilidad. Por lo tanto, para conseguir reguladores LDO dedicados a aplicaciones SoC, sin condensadores de salida externos, se necesita un camino rápido en un LDO completamente integrado para disminuir el tiempo de reacción $\mathrm{y}$, consecuentemente, salvar la limitación de velocidad del lazo de realimentación, asegurando, a su vez, los requerimientos de estabilidad. Una manera simple de llevar a cabo dicho camino que incremente la velocidad, es utilizar un condensador de realimentación entre el nodo de salida y la puerta del transistor de paso $M_{P}$. Si una desviación instantánea se produce en la salida del LDO, dicho condensador actuará como una red diferencial y podrá proporcionar o absorber una corriente considerable a la capacidad de puerta de $M_{P}$ [8]. De acuerdo al efecto Miller, este condensador puede proporcionar una distancia suficientemente elevada entre los polos del sistema antes mencionado. Sin embargo, esta red también crea un camino directo (feedforward) a la salida $\mathrm{y}$, como consecuencia, reduce el margen de fase del circuito al reducirse el valor de la frecuencia del cero en el semiplano derecho (RHP zero), especialmente para bajos valores de la transconductancia $g_{m p}$ del transistor $M_{p}$. Para evitar este problema, puede utilizarse el condensador pero con un buffer de corriente desde la salida al nodo de la puerta de $M_{P}$. En este caso, aparece un cero en el semiplano izquierdo, en lugar de en el semiplano derecho, debido al camino unidireccional desde el nodo de salida a la puerta de $M_{P}$, que ayuda, de forma importante, a mejorar el margen de fase del regulador.

Una forma simple de llevar a cabo el buffer de corriente y el amplificador de error es utilizar un amplificador cascodo, tal y como se muestra en la Fig. 2, donde el transistor $M_{4}$ está en una configuración de puerta común y actúa como buffer de corriente. El modelo en pequeña señal del circuito se representa a su vez en la Fig. 3, donde $R_{1}, R_{o}$, y $R_{b}$ son, respectivamente, la resistencia de salida del amplificador de error, la resistencia equivalente del nodo de salida y resistencia de entrada del buffer de corriente. Además, $g_{m b}$ es la transconductancia del transistor $M_{4}$ que, como ya hemos dicho, actúa de buffer de corriente. $C_{1}$ es la capacidad total en la puerta de $M_{P}$, y $C_{b}$ es el condensador de compensación. Como se aprecia, existen dos lazos de realimentación: el primero o "principal" a través del amplificador de error $g_{m 1}, \mathrm{y}$, el segundo, a través del buffer de corriente $g_{m b}$. Así, la función de transferencia en lazo abierto del circuito viene dada por:

$$
H(s)=\frac{-A_{0}\left(1+s \frac{C_{b}}{g_{m b}}\right)}{\left(1+s C_{b} g_{m p} R_{1} R_{o}\right)\left(1+s \frac{C_{1} C_{o u t}}{C_{b} g_{m p}}+s^{2} \frac{C_{1} C_{o u t}}{g_{m p} g_{m b}}\right)}
$$




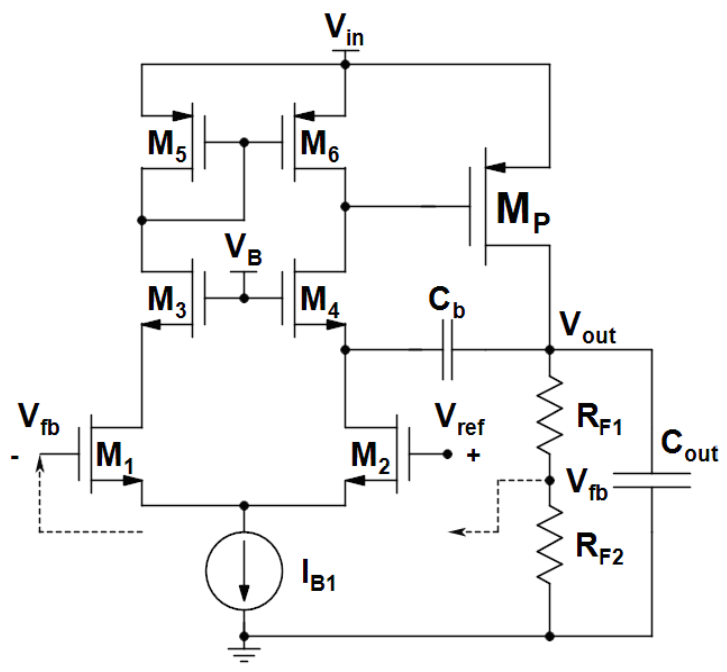

Figura 2. El regulador LDO con el buffer de corriente incluido en el amplificador de error.

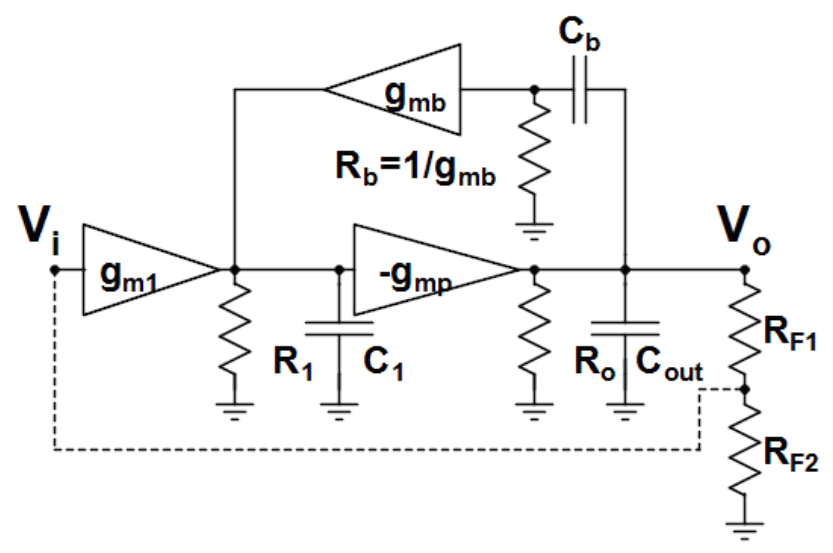

Figura 3. Modelo en pequeña señal del regulador LDO con el buffer de corriente incluido en el amplificador de error.

Como puede verse, la respuesta en lazo abierto del circuito tiene un cero LHP, un polo dominante, y un par de polos complejo-conjugados. La frecuencia natural, $\omega_{n}$, y el factor de amortiguamiento, $\zeta$, de dichos polos complejos vienen dados, respectivamente por:

$$
\omega_{n}=\sqrt{\frac{g_{m p} g_{m b}}{C_{1} C_{\text {out }}}} \text { y } \zeta=\frac{1}{2 C_{b}} \sqrt{\frac{C_{1} C_{\text {out }} g_{m b}}{g_{m p}}}
$$

Modificando la corriente de carga desde 0 a $I_{\max }$, la transconductancia $g_{m p}$ se incrementa y, por lo tanto, los polos complejo-conjugados se desplazan progresivamente a frecuencias mayores. Como consecuencia, el margen de fase se ve incrementado. La Fig. 4 representa la respuesta frecuencial en lazo abierto del regulador LDO para $g_{m 1}=180 \mu S, g_{m 4}=205$ $\mu S, C_{b}=10 p F, R_{F 1}=312 \mathrm{k} \Omega, R_{F 2}=248 \mathrm{k} \Omega$, y $C_{\text {out }}=100 \mathrm{pF}$. Como es obvio, se consigue una separación de polos para los diferentes valores considerados de la corriente de carga. La respuesta transitoria de la carga para un escalón de corriente de 0 a $100 \mathrm{~mA}$ con un tiempo de subida de sólo $1 \mu \mathrm{s}$ y diferentes valores de $C_{b}$ se muestran en la Fig. 5. Como se aprecia, incrementando el valor de $C_{b}$, se incrementa también la capacidad efectiva entre el nodo de salida y la puerta de $M_{P}$. Ello provoca una mejora en las variaciones de la tensión de salida. Sin embargo, como contrapunto, esto también implica un incremento del área de silicio del chip.

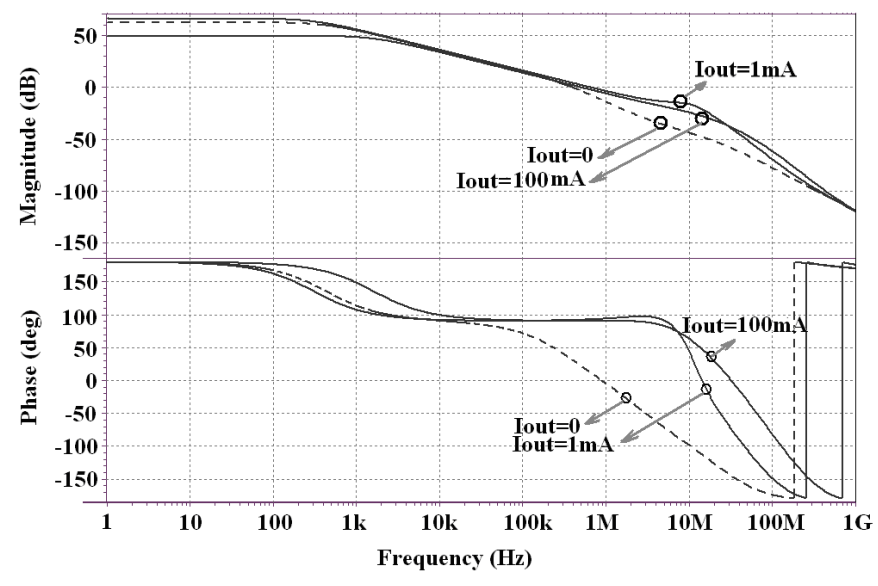

Figura 4. Respuesta en AC y en lazo abierto del regulador LDO con el buffer de corriente incluido.

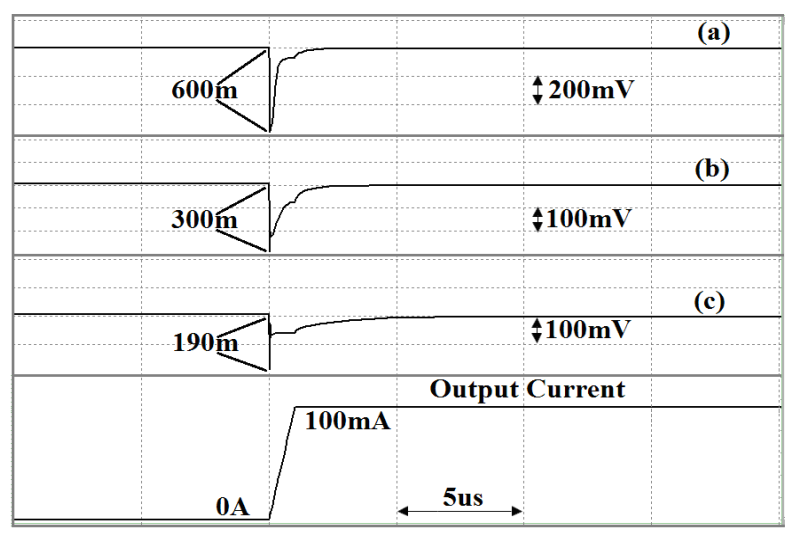

Figura 5. Respuesta transitoria de la tensión de salida del LDO con el buffer de corriente incluido para diferentes capacidades $C_{b}:(a) C_{b}=1 \mathrm{pF},(b) C_{b}=10 \mathrm{pF}, \mathrm{y}$ (c) $C_{b}=100 \mathrm{pF}$.

Una forma efectiva de incrementar el valor del condensador, sin disparar el área necesaria de silicio, es utilizar un multiplicador de capacidad [2, 8]. La Fig. 6 muestra la realización a nivel de circuito de un multiplicador de capacidad basado en amplificador de error [14]. El resistor $R_{b}$ de realimentación paralela-paralela (shunt-shunt) reduce la impedancia de entrada "vista" por el condensador $C_{b}$. La corriente a través de dicho condensador es sensada y convertida en la tensión $V_{b 1} \mathrm{y}$, entonces, reconvertida nuevamente a corriente a través de la etapa de transconductancia $g_{m b 2}$ (implementada por el transistor $M_{2}$ ). El factor de amplificación para la capacidad $C_{b}$ es, aproximadamente, igual al producto $g_{m b 1} R_{b}$, donde $g_{m b 1}$ es la transconductancia del transistor $M_{1}$. Asumiendo que $C_{1}$ es la capacidad parásita en el nodo de drenador de $M_{1}$, la función de transferencia desde la entrada $V_{i}$ al nodo $V_{b 1}$ es igual a:

$$
\frac{V_{b 1}}{V_{i}}=\frac{-s\left(g_{m b 1} R_{b}-1\right) C_{b}}{g_{m b 1}\left(1+s \frac{C_{b}}{g_{m b 1}}+s^{2} \frac{C_{b} R_{b} C_{1}}{g_{m b 1}}\right)}
$$




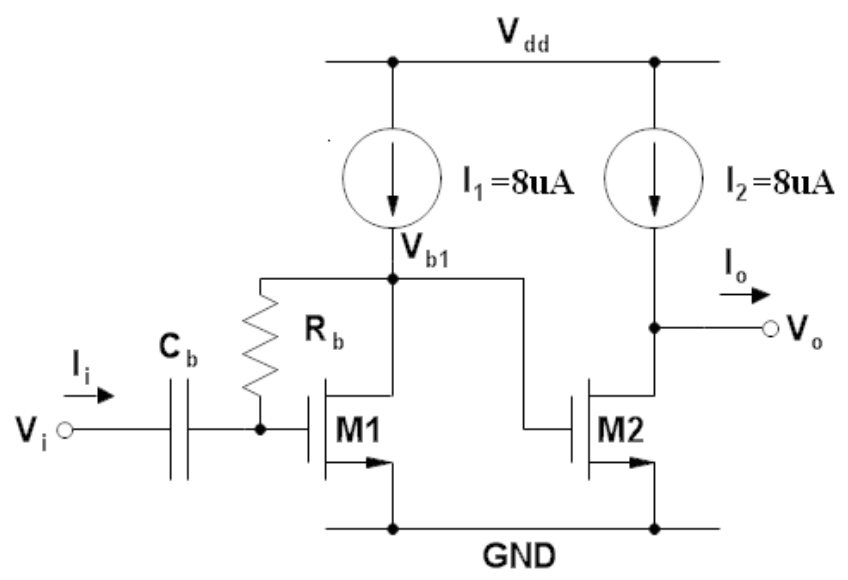

Figura 6. Multiplicador de capacidad basado en amplificador de corriente.

Los polos parásitos del circuito pueden degradar el factor multiplicativo de la capacidad en altas frecuencias. Sin embargo, debido al hecho de que la frecuencia de ganancia unidad del regulador LDO es, generalmente, inferior a $1 \mathrm{MHz}$, estos polos parásitos pueden ser desplazados a frecuencias mayores que dicho valor y, por tanto, la Eq. (5) puede ser simplificada a:

$$
\frac{V_{b 1}}{V_{i}}=\frac{-s\left(g_{m b 1} R_{b}-1\right) C_{b}}{g_{m b 1}}
$$

Este circuito puede ser utilizado como una etapa separada conectada entre el nodo de salida del regulador LDO y la salida del amplificador de error $[2,8,15]$. No obstante, si se realiza esto, se tendrá un mayor consumo de potencia sin ninguna ventaja adicional en el propio lazo de realimentación principal del LDO, como ya se ha comentado previamente. Adicionalmente, en algunos casos, podría incluso ser necesario un tercer lazo para mantener la estabilidad del circuito, que causará, a su vez, un mayor incremento si cabe en el consumo de potencia [15]. Ahora bien, si el multiplicador de capacidad puede ser incluido en el propio amplificador de error, podrá obtenerse un ahorro en el consumo de potencia del circuito.

\section{REgUlAdOR LDO PROPUESTO}

La Fig. 7 muestra el esquemático a nivel de transistores del regulador LDO propuesto, en el que el multiplicador de capacidad ha sido incluido en el propio amplificador de error. Dicho multiplicador consiste en el condensador $C_{b}$, el resistor $R_{b}$, los transistores $M_{6}$ y $M_{8}$, y las fuentes de corriente $M_{5}$ y $M_{7}$. De hecho, estos cuatro transistores forman parte, a su vez, del amplificador de error del LDO.

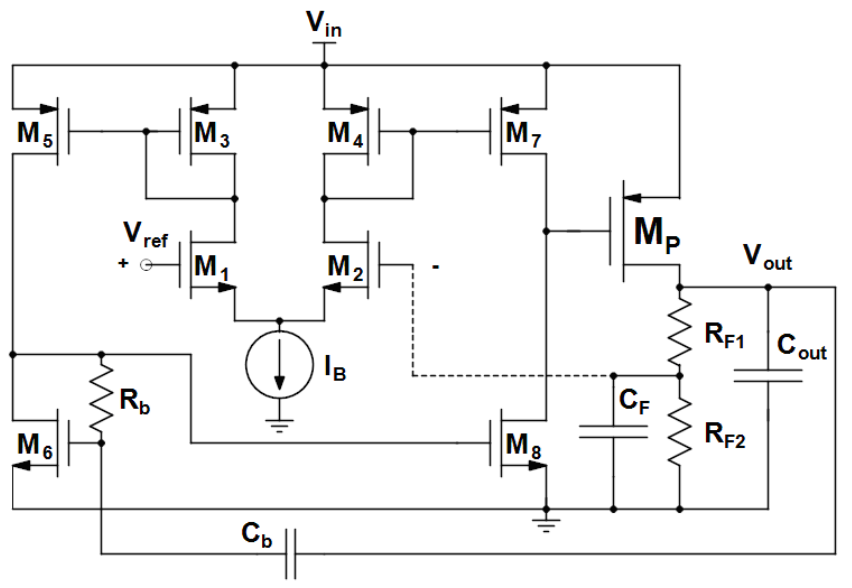

Figura 7. Esquemático a nivel de transistores del regulador LDO propuesto.

La Fig. 8 muestra el modelo en pequeña señal del regulador LDO propuesto, donde $g_{m p}$ y $g_{m b}$ son, respectivamente, la transconductancia de los transistores $M_{P}$ y $\left(M_{6} / M_{8}\right)$. Por otro lado, $R_{\mathrm{o}}, R_{1}$, y $R_{p b}$ son las resistencias equivalentes del nodo de salida, de la puerta de $M_{P}$, y de la puerta de $M_{8}$, respectivamente. $C_{1}$ y $C_{p b}$ son, a su vez, las capacidades equivalentes en la puerta de $M_{P}$ y en la puerta de $M_{8}$, respectivamente. Llevando a cabo el análisis en $\mathrm{AC}$, de una manera similar a como se hace en [16], la función de transferencia en lazo abierto del LDO está dada por la Eq. (7):

$$
\begin{gathered}
H(s)=\frac{A_{0}\left(1+s \frac{\left(g_{m b} R_{b}+1\right) C_{b}}{2 g_{m b}}\right)}{\left(1+\frac{s}{\omega_{p 1}}\right)\left(1+s \frac{C_{1} C_{o u t}}{g_{m p}\left(g_{m b} R_{b}-1\right) C_{b}}\right)}, \\
\frac{\left[1+s \frac{1}{g_{m b} R_{b}+1}\left(R_{b} C_{p b}+\frac{C_{1}}{g_{m p}}\right)\right]}{\left(1+s \frac{C_{b}}{g_{m b}}+s^{2} \frac{C_{b} R_{b} C_{p b}}{g_{m b}}\right)\left(1+s R_{F 2} C_{F}\right)}
\end{gathered}
$$

donde $A_{0}$ y $\omega_{p 1}$ son la magnitud en DC de la ganancia de lazo y el polo dominante del circuito, respectivamente, dados a su vez por:

$$
\begin{aligned}
& A_{0}=g_{m 1} R_{1} g_{m p} R_{o} \frac{R_{F 2}}{R_{F 1}+R_{F 2}} \mathrm{y} \\
& \omega_{p 1}=\frac{1}{\left[R_{1} g_{m p} R_{o}\left(g_{m b} R_{b}-1\right) C_{b}\right]}
\end{aligned}
$$

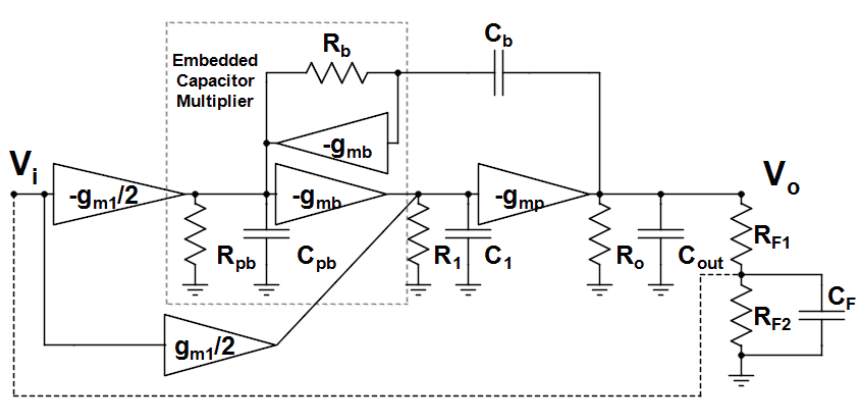

Figura 8. Modelo en pequeña señal del regulador LDO propuesto.

Adicionalmente, la frecuencia de ganancia unidad, el polo no 
dominante, y el cero LHP del circuito debidos a la red rápida creada por $C_{b}$ y $R_{b}$ están definidos, respectivamente, por:

$$
\begin{gathered}
\omega_{T} \approx \frac{g_{m 1} R_{F 2}}{\left[\left(g_{m b} R_{b}-1\right) C_{b}\right]\left[R_{F 2}+R_{F 1}\right]}, \\
\omega_{p 2} \approx \frac{g_{m p}\left(g_{m b} R_{b}-1\right) C_{b}}{C_{1} C_{\text {out }}} \mathrm{y} \\
\omega_{Z 1}=\frac{2 g_{m b}}{\left[\left(g_{m b} R_{b}+1\right) C_{b}\right]}
\end{gathered}
$$

La simulación de la respuesta en frecuencia en lazo abierto del regulador LDO propuesto, para diferentes valores de la corriente de salida, y considerando $R_{b}=100 \mathrm{k} \Omega, R_{F 1}=160 \mathrm{k} \Omega$, $R_{F 2}=200 \mathrm{k} \Omega, C_{b}=2 \mathrm{pF}, C_{F}=1 \mathrm{pF}$, y $C_{\text {out }}=100 \mathrm{pF}$, se muestran en la Fig. 9. Como es obvio, cuando la corriente de carga se incrementa de 0 a $I_{\max }$, el polo dominante y el cero LHP permanecen, aproximadamente, fijos, pero el polo no dominante se desplaza a frecuencias mayores $y$, como consecuencia, el margen de fase mejora. Los valores de margen de fase del LDO diseñado para valores de corriente de salida de $0 \mathrm{~mA}, 1 \mathrm{~mA}$, y $100 \mathrm{~mA}$ son $40^{\circ}, 87^{\circ}$, y $89^{\circ}$, respectivamente.

De acuerdo con la Eq. (7), otro cero LHP y un par de polos complejo-conjugados, todos ellos debidos a los componentes parásitos del multiplicador de capacidad, son creados a frecuencias mayores que la frecuencia $\omega_{T}$. Aunque el cero LHP mejora el margen de fase del sistema, conviene destacar que podría reducir el margen de ganancia del lazo si se sitúa cercano a $\omega_{T}$. Este aspecto, juntamente con los polos complejo-conjugados del sistema, que muestran un pico en la curva de magnitud y una rápida transición en la curva de desfase, puede degradar la estabilidad del sistema [8]. Para evitar este problema, el condensador $C_{F}$ se ha añadido con el objetivo de cancelar el efecto pernicioso del cero creando un polo a frecuencias mayores de $\omega_{T}$.

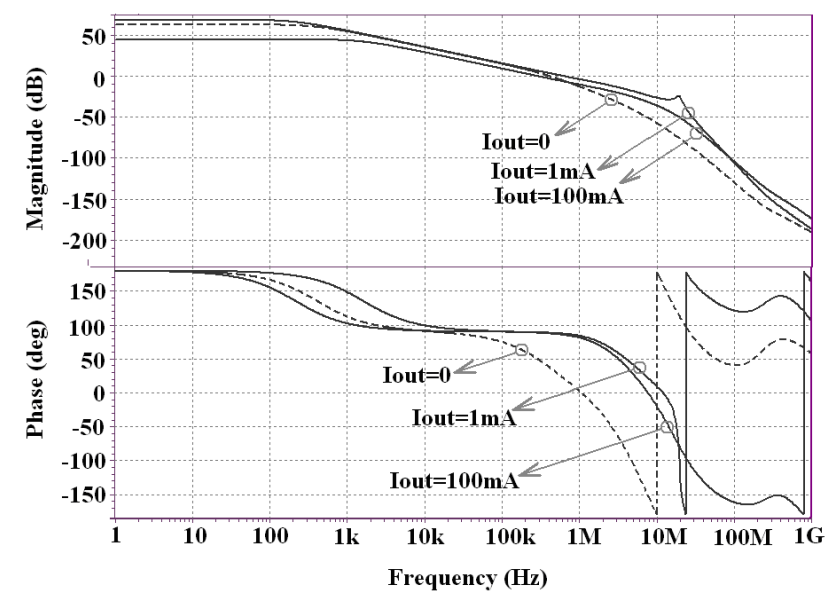

Fig. 9. Respuesta frecuencial en lazo abierto del regulador LDO propuesto.

\section{CARACTERIZACIÓN DEL CIRCUITO}

El regulador LDO propuesto ha sido diseñado y se ha realizado su layout, tal y como muestra la Fig. 10. Las métricas en cuanto a sus prestaciones corresponden a simulaciones postlayout en HSPICE ${ }^{\circledR}$ para una tecnología CMOS de 0,35 $\mu \mathrm{m}$. El área útil requerida para la implementación del chip es de 506x $359 \mu \mathrm{m}^{2}$, de la que el $77 \%$ es ocupado por el condensador de salida de $100 p F$. El LDO se ha diseñado para generar una tensión de salida $V_{\text {out }}=1,8 \mathrm{~V}$ para una tensión de entrada $V_{\text {in }}$ comprendida entre 2 y $3,5 \mathrm{~V}$, con una tensión de referencia $V_{\text {ref }}=1 \mathrm{~V}$, y una corriente de polarización $I_{B}=1 \mu \mathrm{A}$. Mediante este diseño es posible entregar una corriente entre $0 \mathrm{~mA}$ y $100 \mathrm{~mA}$ con un consumo de corriente del circuito en reposo de sólo 22 $\mu A$. La relación de aspecto del transistor de paso es de $W / L=17 \mathrm{~mm} / 0.35 \mu \mathrm{m}$. Dicho transistor cae en su región óhmica para elevados valores de la corriente de salida y, por tanto, para tener suficiente ganancia de lazo para dichas corrientes de salida elevadas, la longitud de los transistores $M_{5}$ a $M_{8}$ es varias veces mayor que la longitud mínima que permite la tecnología. Las diferentes relaciones de aspecto de los transistores del diseño llevado a cabo se muestran en la Tabla I. Por otro lado, algunas especificaciones de diseño se muestran en la Tabla II.

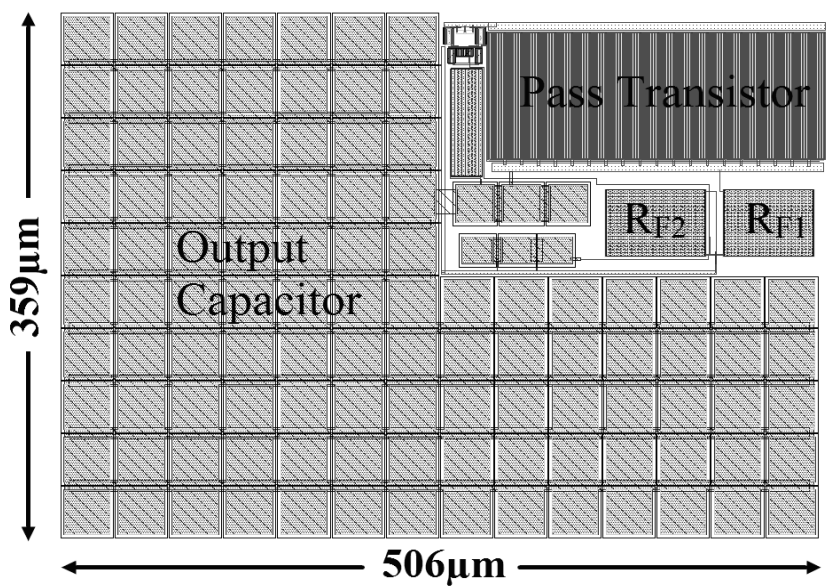

Figura 10. Layout del regulador LDO propuesto.

TABLA I. RELACIÓN DE ASPECTO DE LOS TRANSISTORES DEL REGULADOR LDO PROPUESTO.

\begin{tabular}{|c|c|c|}
\hline Transistores & $\mathbf{W}(\boldsymbol{\mu m})$ & $\mathbf{L}(\boldsymbol{\mu m})$ \\
\hline$M_{1}, M_{2}$ & 4 & 1 \\
\hline$M_{3}, M_{4}$ & 1 & 2.8 \\
\hline$M_{5}, M_{7}$ & 10 & 2.8 \\
\hline$M_{6}, M_{8}$ & 9 & 1.5 \\
\hline$M_{P}$ & 17000 & 0.35 \\
\hline
\end{tabular}

TABLA II. ESPECIFICACIONES DE DISEÑO DEL REGULADOR LDO PROPUESTO.

\begin{tabular}{|c|c|}
\hline Ganancia de lazo & $45-68 d B$ \\
\hline Regulación de Carga & $49 \mu \mathrm{V} / \mathrm{mA}$ \\
\hline Regulación de Línea & $7 \mathrm{mV} / \mathrm{V}$ \\
\hline PSR@1 mA & $-43 \mathrm{~dB} @ 10 \mathrm{kHz}$ \\
\hline
\end{tabular}

Las regulaciones de carga y de línea se muestran en las Fig. 11 y 12, respectivamente. El LDO diseñado permite obtener regulaciones de carga y de línea de $49 \mu \mathrm{V} / \mathrm{mA}$ y $7 \mathrm{mV} / \mathrm{V}$, respectivamente. Por su parte, la Fig. 13 muestra la respuesta transitoria de la tensión de salida del regulador LDO propuesto para diferentes variaciones en la corriente de carga del circuito. Puede observarse que el LDO es estable en todos los casos, y la variación de la tensión de salida, $\Delta V_{\text {out }}$, es siempre inferior a los $200 \mathrm{mV}$. La respuesta transitoria de línea del LDO para una corriente máxima en la carga es mostrada en la Fig. 14, donde 
los cambios de la tensión de línea entre 2 y $3 V$ causan variaciones en la tensión de salida $\Delta V_{\text {out }}$ de solamente $100 \mathrm{mV}$.

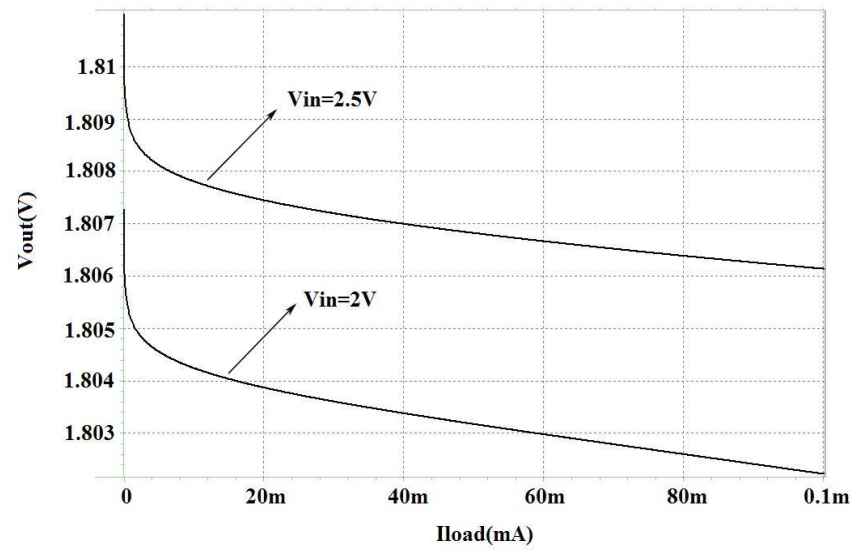

Figura 11. Regulación de carga del regulador LDO propuesto para diferentes valores de la tensión de entrada.

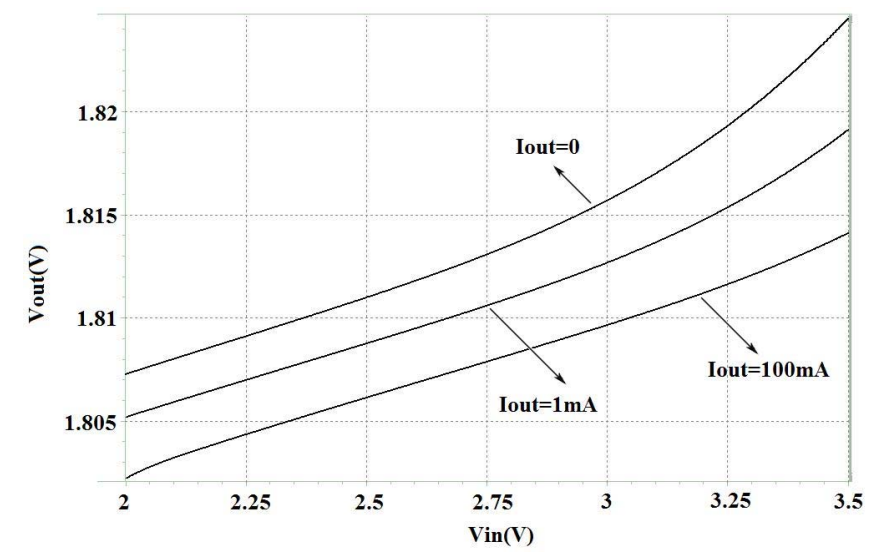

Figura 12. Regulación de línea del regulador LDO propuesto para diferentes valores de la corriente de carga.

Para tener una comparación coherente y poner de manifiesto las ventajas en cuanto a ahorro de potencia de la red de alta velocidad incluida en el regulador LDO propuesto, los LDOs reportados en [8] y [10] han sido también simulados en HSPICE $^{\circledR}$ (juntamente con el propuesto en el presente trabajo) con los parámetros mencionados en los correspondientes artículos. La Fig. 15 muestra la eficiencia de corriente (CE) de los tres reguladores LDO considerados en función de la corriente de carga entre $0 m A$ y $I_{\max }$. En los trabajos [8] y [10], los valores de $I_{\max }$ y $C_{\text {out }}$ son $(50 m A-100 \mathrm{pF})$ y $(50 m A-20$ $p F)$, respectivamente, y para el LDO propuesto en el presente trabajo son $(100 m A-100 p F)$. Como puede verse, el regulador LDO propuesto posee una eficiencia mayor, especialmente para bajas corrientes de carga, que es debida en buena manera al ahorro energético del circuito gracias al multiplicador de capacidad en modo corriente incluido en el circuito.
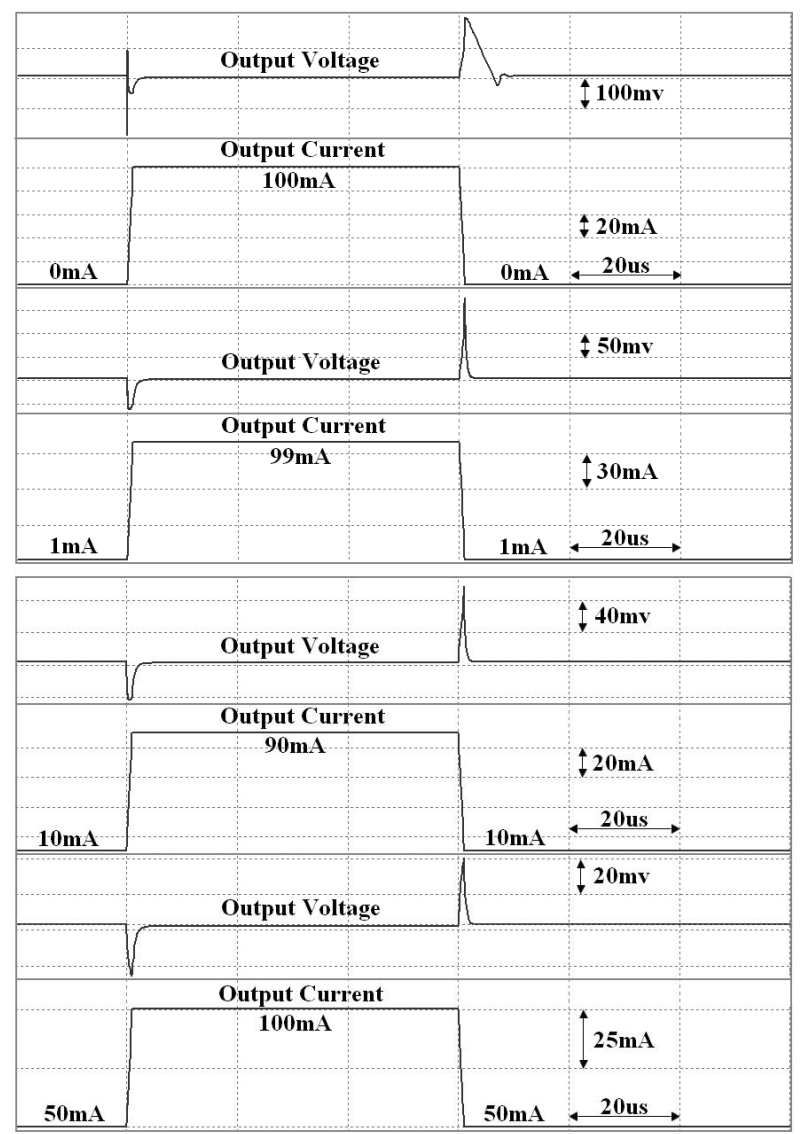

Figura 13. Respuesta transitoria de la tensión de salida del regulador LDO propuesto para diferentes variaciones de la corriente de carga.

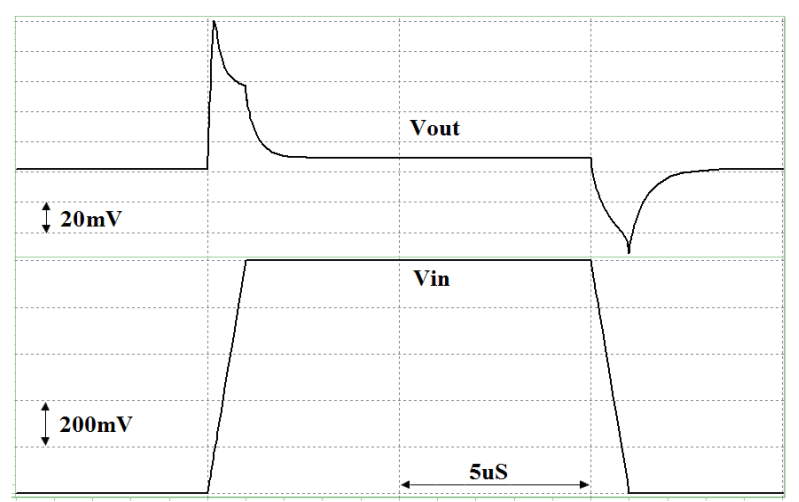

Figura 14. Respuesta transitoria de la tensión de salida del regulador LDO propuesto ante variaciones de la tensión de línea con valor máximo en la corriente de carga.

La Tabla III proporciona un resumen comparativo detallado de las prestaciones de las tres propuestas. Para los reguladores LDO reportados en [8] y [10], se incluyen los resultados tanto experimentales como de simulación (corroborados en el presente trabajo). Para la comparativa se escoge una figura de mérito, FOM, utilizada en [7] (dada por la expresión $\left.F O M=\Delta V_{\text {out }} C_{\text {out }} I_{Q} / I_{\text {out }, \text { max }}^{2}\right)$, que permite comparar la respuesta transitoria de los diferentes LDOs. Un valor menor de FOM implica una mejor respuesta transitoria conseguida por el LDO en cuestión. Como se aprecia en dicha Tabla III, el parámetro 
FOM de la propuesta del presente artículo es inferior que las mostradas en las otras dos propuestas, cosa que indica la capacidad de ahorro energético del multiplicador de capacidad incluido en el chip manteniendo, al mismo tiempo, unas prestaciones dinámicas aceptables ante variaciones de carga.

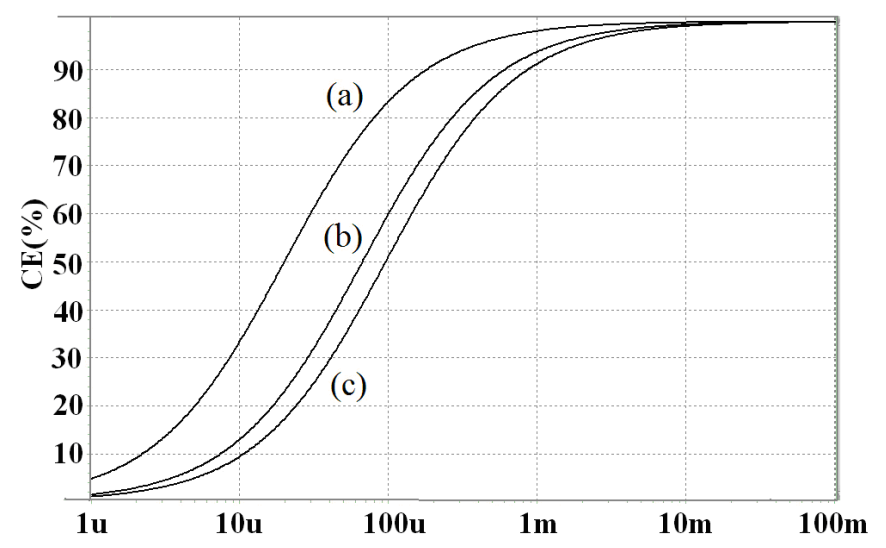

Figura 15. Curvas de eficiencia de corriente (current efficiency, CE) en función de la corriente de carga: $(a)$ para el regulador LDO propuesto en el presente trabajo, (b) para el LDO presentado en [8], y, (c) para el regulador LDO presentado en [10].

TABLA III. COMPARACIÓN DE PRESTACIONES PARA LOS REGULADORES LDO CONSIDERADOS EN EL PRESENTE TRABAJO.

\begin{tabular}{|c|c|c|c|c|c|}
\hline Parámetro & $\begin{array}{c}{[8]} \\
\text { Exp. }\end{array}$ & $\begin{array}{c}{[8]} \\
\text { Sim. }\end{array}$ & $\begin{array}{c}{[10]} \\
\text { Exp. }\end{array}$ & $\begin{array}{c}{[10]} \\
\text { Sim. }\end{array}$ & $\begin{array}{c}\text { LDO } \\
\text { Propuesto }\end{array}$ \\
\hline $\begin{array}{c}\text { Tecnología } \\
(\mu \mathrm{m})\end{array}$ & 0.35 & 0.35 & 0.35 & 0.35 & $\mathbf{0 . 3 5}$ \\
\hline$V_{\text {in }}(V)$ & 3 & 3 & 1.2 & 1.2 & $\mathbf{2}$ \\
\hline$V_{\text {out }}(V)$ & 2.8 & 2.8 & 1 & 1 & $\mathbf{1 . 8}$ \\
\hline$I_{\text {out }}(m A)$ & $0-50$ & $0-50$ & $0-50$ & $0-50$ & $\mathbf{0 - 1 0 0}$ \\
\hline $\begin{array}{c}\text { Corriente } \\
\text { de reposo } \\
(\mu A)\end{array}$ & 65 & 66 & 95 & 95 & $\mathbf{2 2}$ \\
\hline$C_{\text {out }}(p F)$ & 100 & 100 & $>20$ & 20 & $\mathbf{1 0 0}$ \\
\hline$T_{\text {settle }}(\mu \mathrm{S})$ & 15 & 4 & 0.3 & 1.4 & $\mathbf{8 . 5}$ \\
\hline$\Delta V_{\text {out }}(\mathrm{m} V)$ & 90 & 110 & 180 & 200 & $\mathbf{2 0 0}$ \\
\hline $\begin{array}{c}C E(\%) \text { en } \\
\text { condiciones } \\
\text { de carga } \\
\text { máxima }\end{array}$ & 99.87 & 99.86 & 99.81 & 99.81 & $\mathbf{9 9 . 9 7}$ \\
\hline FOM $(f s)$ & 234 & 290 & 136 & 152 & $\mathbf{4 4}$ \\
\hline
\end{tabular}

\section{CONCLUSIONES}

El presente artículo ha presentado un regulador LDO CMOS completamente integrado, y sin condensador de salida off-chip, basado en un multiplicador de capacidad en modo corriente incluido en el lazo de realimentación. Se ha mostrado que, incluyendo dicho multiplicador dentro del propio amplificador de error del LDO, no solamente podemos incrementar las prestaciones dinámicas del circuito ante variaciones de carga, garantizando, al mismo tiempo, la estabilidad del sistema, sino que también podemos reducir la potencia consumida por el circuito. El LDO propuesto ha sido diseñado y se han llevado a cabo simulaciones post-layout en HSPICE $^{\circledR}$ para un proceso CMOS de 0,35 $\mu \mathrm{m}$, con el fin de obtener una tensión de salida de 1,8 $\mathrm{V}$. La variación máxima de la tensión de salida es igual a $200 \mathrm{mV}$ para valores de la corriente de carga que oscilan entre $0 m A$ y $100 m A$. Los resultados de comparación basados en el parámetro $F O M$ ponen de manifiesto las prestaciones del regulador LDO propuesto.

\section{AgRADECIMIENTOS}

Este trabajo ha sido parcialmente financiado por el Gobierno Español, a través del Ministerio de Ciencia e Innovación, gracias al proyecto TEC2010-15765 (subprograma MIC), y del Ministerio de Economía y Competitividad, gracias al proyecto DPI2013-47799-C2-2-R.

\section{REFERENCIAS}

[1] A. Saberkari, Herminio Martinez, and E. Alarcon, "Fast Transient Response CFA-Based LDO Regulator," in Proc. IEEE Int. Symp. Circuits Syst. (ISCAS'12), May 2012, pp. 3150-3153.

[2] C. K. Chava and J. Silva-Martinez, "A Frequency Compensation Scheme for LDO Voltage Regulators," IEEE Trans. Circuits Syst. I, vol. 51, no. 6, pp. 1041-1050, Jun. 2004.

[3] M. Al-Shyoukh, H. Lee, and R. Perez, "A Transient-Enhanced LowQuiescent Current Low-Dropout Regulator with Buffer Impedance Attenuation," IEEE J. Solid-State Circuits, vol. 42, no. 8, pp. 1732-1742, Aug. 2007.

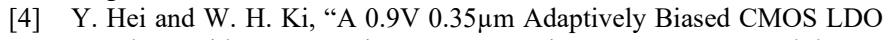
Regulator with Fast Transient Response," in Proc. IEEE Int. Solid-State Circuits Conf. (ISSCC'08), Dig. Tech. Papers, Feb. 2008, pp. 442-443.

[5] K. N. Leung and Y. S. Ng, "A CMOS Low-Dropout Regulator with a Momentarily Current-Boosting Voltage Buffer," IEEE Trans. Circuits Syst. I, vol. 57, no. 9, pp. 2312-2319, Sept. 2010.

[6] M. Ho, K. N. Leung, and K. L. Mak, "A Low-Power Fast-Transient 90nm Low-Dropout Regulator with Multiple Small-Gain Stages," IEEE J. Solid-State Circuits, vol. 45, no. 11, pp. 2466-2476, Nov. 2010.

[7] P. Hazucha, T. Karnik, B. Bloechel, C. Parsons, D. Finan, and S. Borkar, "Area-Efficient Linear Regulator with Ultra-Fast Load Regulation," IEEE J. Solid-State Circuits, vol. 40, no. 4, pp. 933-940, Apr. 2005.

[8] R. J. Milliken, J. Silva-Martinez, and E. Sanchez-Sinencio, "Full On-Chip CMOS Low-Dropout Voltage Regulator," IEEE Trans. Circuits Syst. I, vol. 54, no. 9, pp. 1879-1890, Sept. 2007.

[9] W. J. Huang and S. I. Liu, "Capacitor-Free Low Dropout Regulators Using Nested Miller Compensation with Active Resistor and 1-bit Programmable Capacitor Array," IET Circuits Devices Syst., vol. 2, no. 3, pp. 306-316, 2008.

[10] T. Y. Man, K. L. Leung, C. Y. Leung, P. K. T. Mok, and M. Chan, "Development of Single-Transistor-Control LDO Based on Flipped Voltage Follower for SoC," IEEE Trans. Circuits Syst. I, vol. 55, no. 5, pp. 1392-1401, Jun. 2008.

[11] P. Y. Or and K. N. Leung, "An Output-Capacitorless Low-Dropout Regulator with Direct Voltage-Spike Detection," IEEE J. Solid-State Circuits, vol. 45, no. 2, pp. 458-466, Feb. 2010.

[12] J. Guo and K. N. Leung, "A 6- $\mu \mathrm{W}$ Chip-Area-Efficient OutputCapacitorless LDO in 90-nm CMOS Technology," IEEE J. Solid-State Circuits, vol. 45, no. 9, pp. 1896-1905, Sept. 2010.

[13] J. Falin, "ESR, Stability, and the LDO Regulator," Application Report, Texas Instruments Inc., Literature Number: SLVA115, May 2002.

[14] Z. Yan, L. Shen, Y. Zhao, and S. Yue, "A Low-Voltage CMOS LowDropout Regulator with Novel Capacitor-Multiplier Frequency Compensation," in Proc. IEEE Int. Symp. Circuits Syst. (ISCAS'08), May 2008, pp. 2685-2688.

[15] A. Garimella, M. W. Rashid, and P. M. Furth, "Reverse Nested Miller Compensation Using Current Buffers in a Three-Stage LDO," IEEE Trans. Circuits Syst. II, vol. 57, no. 4, pp. 250-254, Apr. 2010.

[16] Z. Yan, "Two-Stage Large Capacitive Load Amplifier with Embedded Capacitor-Multiplier Compensation," in Proc. IEEE Int. Symp. Circuits Syst. (ISCAS'09), May 2009, pp. 2481-2484. 


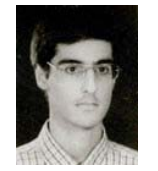

Alireza Saberkari (S'09-M'11) received the B.Sc. degree in Electrical Engineering from Iran University of Science and Technology (IUST), Tehran / University of Guilan, Rasht, Iran, in 2002 and the M.Sc. and Ph.D. degrees both in Electrical Engineering from Iran University of Science and Technology (IUST), Tehran, Iran, in 2004 and 2010, respectively (all with honors). Since 2010, he has been with the Department of Electrical Engineering at University of Guilan as an Assistant Professor. During the period 2008-2009, he joint the group of Energy Processing Integrated Circuits (EPIC), Department of Electronics Engineering, Technical University of Catalunya (UPC), Barcelona, Spain, as a Visiting Scholar and worked on "CMOS Linear LowDropout Regulators for Wideband-Tracking Linear-Assisted Scheme" \& "RF Transmitter Architectures Considering Wideband Adaptive Supply of RF PA". $\mathrm{He}$ has authored or co-authored more than 50 international scientific publications including journals and conference proceedings. He was the technical program committee (TPC) member of the IEEE Latin American Symposium on Circuits and Systems (LASCAS'13, LASCAS'14, and LASCAS'15) and IEEE International Conference on Emerging Technologies and Factory Automation (ETFA'14), and also the organizing committee member of the IEEE International Conference on Pattern Recognition and Image Analysis (IPRIA'15). He has served as a reviewer for the IEEE Transactions on Electron Devices, Electronics Letters, Analog Integrated Circuits and Signal Processing, Wiley International Journal of Circuit Theory and Applications, International Journal of Electronics, Elsevier Microelectronics Journal, Elsevier integration, the VLSI Journal, Journal of Circuits, Systems, and Computers, Electronics and Electrical Engineering, International Journal for the Computation and Mathematics in Electrical and Electronic Engineering, Journal of Low Power Electronics, International Journal of Signal and Data Processing, Iranian Journal of Electrical and Computer Engineering, and also ISCAS, MWSCAS, ICECS, LASCAS, ECCTD, ETFA, and ISWTA conferences. His fields of interest include the areas of Analog, RF, and Mixed-Signal Microelectronics with particular interest in On-Chip Power Management Circuits, Analog Circuits for Energy Harvesting Applications and Biomedical Implants, Linear and Low-Dropout Regulators, Current-Mode Circuit Design, CMOS LNAs and Mixers, RF Power Amplifiers, and Low-Power and Low-Voltage Integrated Circuits. Dr. Saberkari is a member of IEEE Solid-State Circuits and Circuits and Systems societies.

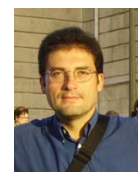

Herminio Martínez-García Herminio received the B.Eng. degree (National Award) in Electrical Engineering, the M.S. degree (National Award) in Electronics Engineering and the Ph.D. degrees in Electronics Engineering (all three with honors) from the Technical University of Catalonia (UPC, BarcelonaTech) in Barcelona, Spain, in 1994, 1998 and 2003, respectively. During the period 1995-1998, Dr. Martinez-Garcia was a halftime Assistant Professor at the Department of Electronics of the College of Industrial Engineering of Barcelona (EUETIB-CEIB), where he became a fulltime Assistant Professor at the same Department in September 1998. In September 2000 he joined the Department of Electronics Engineering of the UPC-BarcelonaTech, where he became an Associate Professor in 2006 and researcher with the Energy Processing and Integrated Circuits (EPIC) Group of the UPC. From October 2008 to March 2009, he was a Visiting Professor at the Analog \& Mixed Signal Center (AMSC) of the Department of Electrical and Computer Engineering of the Texas A\&M University (TAMU) at College Station, Texas (USA). Professor Martinez-Garcia currently teaches analog circuits design, communication systems, and data acquisition and control systems. His research focuses on the area of DC-DC power converters and their control, and analog circuit design with emphasis in analog microelectronics and particular interest in continuous-time filters and automating tuning design, analog and energy processing circuits for energy harvesting. He has participated in five Spanish national research projects. He has authored or co-authored about sixty scientific papers in journals and conference proceedings and 15 books and book chapters. Dr. Martinez is a member of the IEEE Solid-State Circuits, Power Electronics, and Education Societies. He has been organizing committee member and technical program committee (TPC) member of different IEEE Conferences such as ETFA 2014 and COMPENG 2014. In addition, he has served as a reviewer for different Journals, Transactions, and Conferences such as Electronics Letters, Analog Integrated Circuits and Signal Processing, Elsevier Integration, the VLSI Journal, and also ISCAS, MWSCAS, ICECS, LASCAS, ECCTD, ETFA, etc.

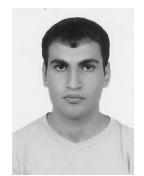

Rasoul Fathipour received the B.Sc. degree in Electrical Engineering from Urmia University, Urmia, Iran in 2010 and the M.Sc. degree in Electrical Engineering from the University of Guilan, Rasht, Iran, in 2012. His fields of interest include the areas of Analog and Mixed-Signal Microelectronics.

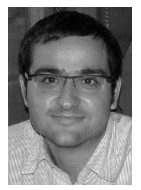

Eduard Alarcón-Cot received the M.Sc. (national award) and $\mathrm{Ph} . \mathrm{D}$. degrees in Electrical Engineering from the Technical University of Catalunya (UPC, BarcelonaTech), Spain, in 1995 and 2000, respectively. Since 1995 he has been with the Department of Electronic Engineering at UPC, where he became Associate Professor in 2000 and is also now co-director of the N3cat center. From August 2003 to January 2004, July-August 2006 and JulyAugust 2010 he was a Visiting Professor at the CoPEC center, University of Colorado at Boulder,US, and during January-June 2011 he was Visiting Professor at the School of ICT/Integrated Devices and Circuits, Royal Institute of Technology (KTH), Stockholm, Sweden. He has co-authored more than 150 international scientific publications, 3 books, 4 book chapters and 4 patents, and has been involved in different National, European and US R\&D projects within his research interests including the areas of analog and mixed-signal integrated circuits, on-chip power management circuits, energy harvesting and wireless energy transfer, and nanotechnology-enabled communications. He has given 18 invited or plenary lectures and tutorials in Europe, America and Asia, was appointed by the IEEE CAS society as distinguished lecturer for 2009-2010 and lectures yearly MEAD courses at EPFL. He is elected member of the IEEE CAS Board of Governors (2010-2013) and member of the IEEE CAS long term strategy committee. He was recipient of the Myril B. Reed Best Paper Award at the 1998 IEEE Midwest Symposium on Circuits and Systems. He was the invited co-editor of a special issue of the Analog Integrated Circuits and Signal Processing journal devoted to current-mode circuit techniques, and a special issue of the International Journal on Circuit Theory and Applications. He coorganized two special sessions related to on-chip power management at IEEE ISCAS03 and IEEE ISCAS06 and a tutorial at IEEE ISCAS09 and ESSCIRC 2011. He was the 2007 Chair of the IEEE Circuits and Systems Society Technical Committee of Power Systems and Power Electronics Circuits. He was the technical program co-chair of the 2007 European Conference on Circuit Theory and Design - ECCTD07, tutorial co-chair at ICM 2010, track co-chair of the IEEE ISCAS 2007, IEEE MWSCAS07, IEEE ISCAS 2008, ECCTD'09, IEEE MWSCAS09, IEEE ICECS'2009, ESSCIRC 2010, PwrSOC 2010, and TPC member for IEEE WISES 2009, WISES 2010, IEEE COMPEL 2010, IEEE ICECS 2010, IEEE PRIME 2011 and ASQED 2011. He served as an Associate Editor of the IEEE Transactions on Circuits and Systems - II: Express briefs (2006-2007) and currently serves as Associate Editor of the Transactions on Circuits and Systems - I: Regular papers (2006-), Elsevier's Nano Communication Networks journal (2009-) and in the Senior Editorial Board of the IEEE Journal on IEEE Journal on Emerging and Selected Topics in Circuits and Systems (2010-). 